\title{
"Goat-Sheep-Mixed-Sign" in Lhasa - Deaf Tibetans' language ideologies and unimodal codeswitching in Tibetan and Chinese sign languages, Tibet Autonomous Region, China
}

\section{Introduction}

Among Tibetan signers in Lhasa, there is a growing tendency to mix Tibetan Sign Language (TSL) and Chinese Sign Language (CSL). I have been learning TSL from deaf TSL teachers and other deaf, signing Tibetan friends since 2007, but in more recent conversations with them I have been more and more exposed to CSL. In such contexts, signing includes not only loan signs, loan blends or loan translations from CSL that have been used in TSL since its emergence, such as signs for new technical inventions or scientific terms. It also includes codeswitching to CSL lexical items related to core social acts, kinship terms or daily necessities, for which TSL has its own signs, such as for concepts including "to marry", "mother", "father”, “teacher”, "house”, “at home”, “real”, "fake”, “wait”, “why”, "thank you" and so on. ${ }^{1}$

Some Tibetan signers refer to the resulting mixed sign language as "neithergoat-nor-sheep sign" (in Tibetan ra-ma-luk lak-da). This phrase is partly derived from the standard Lhasa Tibetan expression of something or somebody being "neither-goat-nor-sheep" (in Tibetan ra-ma-luk), an expression widely used in the context of codeswitching between Lhasa Tibetan and Putunghua (i.e. standard Chinese) and the resulting "neither-goat-nor-sheep language” (in Tibetan

1 Although the acronym TSL is also used for Taiwan Sign Language and Thai Sign Language, I use it here, because it is used in the English designations of many TSL-related publications written in the Tibetan language, co-authored by deaf Tibetans. "Tibetan Sign Language," or TSL, to be sure, is an outsider's term (none of the deaf Tibetans in Lhasa speak, read or write English). It is used in these publications to render the Tibetan terms onkug lakda (TDPF \& HI 2002) or bökyi lakda (TDA 2011), the first literally meaning 'deaf and mute hand signs', the second, 'Tibetan hand signs'. These Tibetan terms do not incorporate the regular Tibetan word for language ( $k e ́$ ), which in its narrow meaning only refers to spoken languages due to its root denoting vocal sounds and utterance. In TSL, bökyi lakda, is signed with the TSL signs BÖ/BÖPA (TIBET/TIBETAN) followed by LAK-DA $(S I G N)$ (which is identical to the one used in International Sign). When this chapter refers to TSL, it only refers to its Lhasa variety.

Ә Open Access. ( 2020 Theresia Hofer, published by De Gruyter. $\Theta$ Br-NC-ND This work is licensed under the Creative Commons Attribution-NonCommercial-NoDerivatives 4.0 License. 
ké ra-ma-luk, Yeshe 2008). Recently, signers have come up with new signs for their own mixing practices, such as "goat-sheep-mixed-sign", "mixed sign" and "Chinese-Tibetan-mixed sign". ${ }^{2}$ The various terms used to name this mixing phenomenon suggest different attitudes toward TSL, CSL, and the practice of mixing.

Based on participant observation, linguistic autobiography interviews (Pavlenko 2007) and many video recorded conversations with and of 25 deaf Tibetan signers over four months of fieldwork in Lhasa during 2016 and 2017 (see Figure 1), this chapter describes several ways that signers learn and mix TSL and CSL and it analyses how Tibetan signers of both TSL and CSL think about this sort of language co-existence. It also relates these sign language practices and ideologies to spoken language practices and ideologies in Lhasa today. ${ }^{3}$

2 There is no widespread written form for sign languages in general, or TSL in particular. When I paraphrase my interlocutors' signing into written text based on notes I took during fieldwork, I use italics within quotation marks. When I translate their signing from video or drawings of, or very detailed notes on, particular signs, either directly into English or into Standard Tibetan and then English, I use quotation marks but no italics. When referring to standard TSL signs, I capitalise the closest Tibetan rendering of that sign, e.g. BÖ, which is followed by an English translation in italics and in square brackets [TIBET]. Except for the titles of books in the references (where I use the full Wylie transcription), I otherwise use phonetically-based spelling for Tibetan terms following the Tibetan and Himalayan Library, 2010 Online Tool (http://www.thlib.org/reference/transliteration/phconverter.php), and italicise these. For Chinese, I use pinyin transliteration and indicate Chinese terms with the use of C. in front of them. For CSL I follow the same methods as for paraphrasing and translating my interlocutors who use TSL, as outlined above, and do the same in those instances when these languages are mixed.

3 This chapter is part of a research project (funded by the Wellcome Trust, Grant 104523), about the lives and signing practices of deaf Tibetans in contemporary Lhasa, capital of the Tibet Autonomous Region (TAR). I refer to the research participants as signers, given that sign language is their preferred communication mode. In line with recent trends (e.g. Kusters and Friedner 2015), I use deaf with a lowercase "d" as a more encompassing category than "Deaf" or "d/Deaf", which in earlier work in Deaf Studies was used to highlight socio-cultural identity and/or the mixed nature of audiological and socio-cultural conditions. 


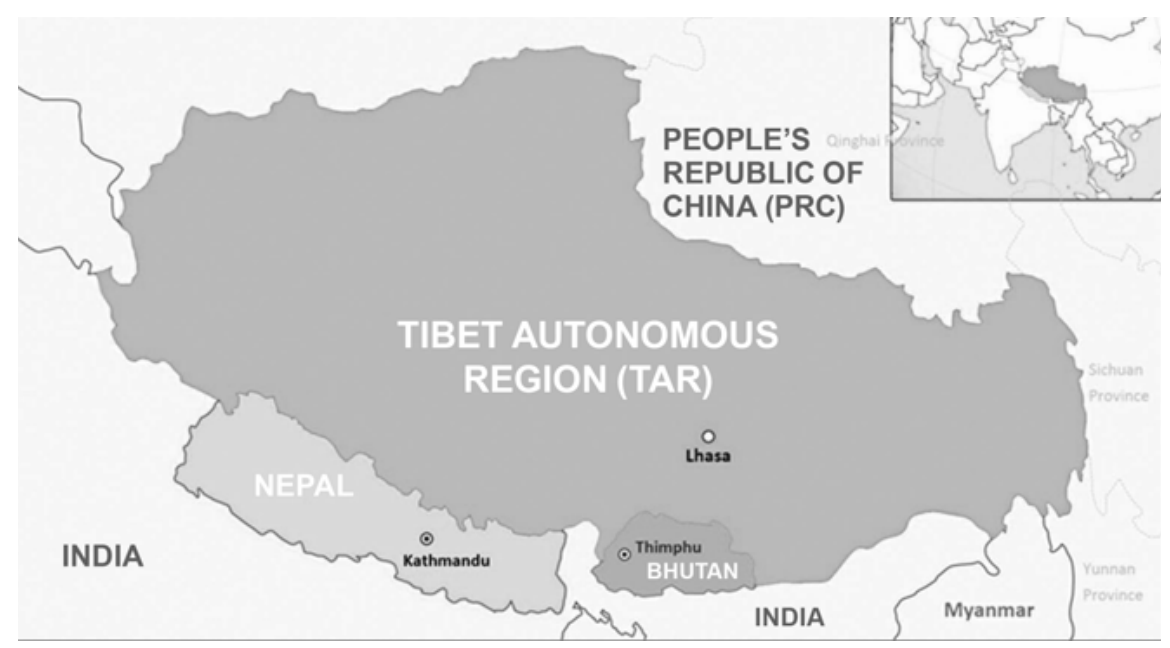

Figure 1. Map of the Tibet Autonomous Region and Lhasa within the People's Republic of China.

In this chapter I use Kroskrity's broad definition of language ideology, as a "ubiquitous set of diverse beliefs, however implicit or explicit they may be, used by speakers of all types as models for constructing linguistic evaluations and engaging in communicative activity" (2004: 497). To this need to be added also beliefs and attitudes towards different linguistic modalities and the specific possibilities they may offer its users. While language ideologies have been explored with regard to distinct signed languages (Kusters 2014, Reagan 2010), attitudes towards and practices of mixing and co-usage of sign languages are the focus of a newly emerging field of research (Adam 2015, Plaza-Pust and Morales-López 2008, Quinto-Pozos 2000, Zeshan and Panda 2015). ${ }^{4}$ This article contributes to the documentation and analysis of how people codeswitch ${ }^{5}$ between two sign languages, and analyses attitudes about such mixing. It also points out a phenomenon which TSL signers call "spontaneous sign."

4 Noteworthy here is the five-year research project on sign multilingualism that was carried out at the University of Central Lancashire, UK. http://www.uclan.ac.uk/research/explore/projects/multilingual_behaviours_sign_language_users.php

5 I follow Crystal's definition of codeswitching, as "The use by a speaker of more than one language, dialect or variety during a conversation. Which form is used depends on factors such as the nature of the audience, the subject matter, and the situation in which the conversations take place.” (1992: 69-70), and Myers-Scotton's distinction between loans and codeswitching as being one mainly of frequency and predictability: while the use of loan words is to some extent predictable, codeswitching is not (1997: 191-207). 
The chapter starts by offering some background information on the recent emergence of TSL, its users and their social and political context in Lhasa; the development of the Lhasa Special School since its foundation in 2000 and the role of Chinese Sign Language; the phenomenon of TSL-CSL codeswitching and that of "spontaneous sign". The core of the chapter presents my findings from Lhasa and an analysis in terms of three broad ideologies about unimodal codeswitching: language purism and language-related pragmatism among the TSL-dominant signers, and linguistic hierarchies among CSL-dominant Tibetan signers. I discuss each of these three language ideologies in relation to local categories used by the signers: The "neither-goat-nor-sheep sign language" I relate to ideologies of language purism they have expressed, with considerable hesitation about code-switching implied. The concept of the "goat-and-sheep-sign" or as one informant put it "a bird with two sign heads" on the other hand values an "and-and" perspective and I relate this to other expressions of a pragmatic ideology about language mixing. Last, CSL-dominant signers have two new signs for the phenomenon of mixing that of RA-LUK-LAK-DRÉ (GOAT-SHEEP-SIGN-MIXED) and of GYA-BÖ-LAK-DRÉ (CHINESE-TIBETAN-SIGN-MIXED). These I relate to ideas that were have expressed about linguistic hierarchies between the national (CSL) and the local (TSL) sign language.

\section{The recent emergence of Tibetan Sign Language, its users and the social and political context}

While a range of communication practices surely existed among deaf people and between deaf and hearing people before 2000, from that year onwards significantly new communication opportunities developed among deaf Tibetans in Lhasa. That year the International NGO Handicap International (HI) started working with deaf Tibetans and established a project to formalize and support what they called "Tibetan Sign Language" (or for short TSL) through the production of sign language dictionaries and Deaf Club activities (Hofer 2017; Hofer and Sagli 2017; TDA 2011; TDPF \& HI 2002, 2005). The project also created a new and at first two-handed TSL manual alphabet that later become one-handed (TDPF \& HI 2002: 16, TDA 2005: 106). In 2004 the Tibet Deaf Association (TDA) was formed with logistical and financial support from HI, backed and supervised by the Tibet Disabled People's Federation (TDPF), the TAR branch of the China-wide, governmental China Disabled People's Federation (CDPF). The various activities by and 
for deaf Tibetans (partly organized and partly funded by HI), were a Sunday Deaf Club; meetings to collect, document and standardize signs; and TSL courses in Lhasa and in selected nearby county towns. In 2014 Handicap International had to stop working in Tibet as their cooperation contract was not renewed by the government. The TDA's work has since been under direct TDPF management, effectively becoming a government organization, with no input from a non-governmental organization. Many of its prior activities came to a halt or are being carried out with greater difficulty. While deaf activists in China proper ${ }^{6}$ are able to defend regional sign varieties and dialects of CSL, and sometimes also TSL, deaf Tibetans and the TDA find in Lhasa and the Tibet Autonomous Region (TAR) it is increasingly difficult to carry on TSL-related activities. In their politically highly charged environment they often fear repercussions if officials and TDPF superiors interpret activities as "politically sensitive," that is, if they are construed as contested aspects of the Tibetan identity and language nexus. As a result, there have only been sporadic TSL courses between 2014 and 2017, and as of July 2017 the production of further TSL language materials had come to an apparent halt, which the TDA lamented.

TSL initially emerged among those adult deaf Tibetans who were involved with the TSL project and the Sunday Deaf Club, beginning in the early 2000s. Most of them had not been formally educated, though some who became deaf as teenagers, had attended regular schools until then. Among this group long-term friendships and romantic relationships developed and TSL was the main language, eventually spreading to a group that I estimated in 2017 to number about 200 to 300. This took place in a context of infrastructure-led, large scale urban growth of Lhasa with accompanying labor migration from rural to urban areas. A majority of people joining the TDA activities were therefore initially not from Lhasa, but hailed from different places across the TAR. Following anthropologist Friedner, it would be apt to say that several of those who joined the TDA meetings took "deaf turns", becoming increasingly oriented towards other deaf instead of hearing people, creating deaf selves and deaf sociality (2015: 2).

\footnotetext{
6 When Tibetans speak about the non-Tibetan areas of China, they tend to use the term Gya or Gyanak (Tibetan for "China”). However, when speaking or writing Chinese, the term neidi (Ch. "interior") is commonly used among Tibetans, in many publications translated as "mainland China". I use "China proper" or the "interior" as translation for neidi, but "China" for the Tibetan Gyanak so as to preserve the strong sense of many Tibetans that "Tibet" is a categorically different place from "China”, even though they have been politically absorbed into the People's Republic of China.
} 


\section{The arrival of Chinese Sign Language and the Lhasa Special School}

In 2000, the first government "Special School” - effectively a deaf boarding school but later also housing a small number of children who are blind or with physical or mental disabilities - was established not far from the city center of Lhasa, a city at that time on the brink of large-scale urban transformation. There were initially six Tibetan teachers and one Chinese teacher (all hearing), speaking a combination of Putunghua and Tibetan as well as using what might be called rudimentary "special education-variety CSL" with their students. These teachers had learned that form of CSL in a module on special education, during a one-year paid training stint at the Beijing Municipality Teachers Training School (Bei jing shi fan xue xiao). They had no other exposure to signed communication or to deaf Chinese people.

The first director of the Special School was a Han Chinese man, who had been working in Lhasa as a teacher and who was married to a Tibetan woman. After his son became deaf in early childhood and having considered the very limited educational opportunities that he would encounter in Lhasa, he petitioned the local authorities to establish the first local Special School and succeeded.

For the first batch of students in 2000, the Lhasa Special School management could only recruit seven deaf Tibetan children via Lhasa neighborhood committees. Over the years, as word spread and the school grew, more and more students came to attend, also from other parts of the TAR. In 2017 the school was home to over 160 deaf students aged between 7 and 21 and four more Special Schools had in the meantime been established in other locations in the TAR.

The Lhasa Special School offers the 9 years of obligatory education, divided into 6 years of primary school and 3 years of lower middle school. In 2017, plans were made to start in the near future also a high school (i.e. upper middle school) within the Lhasa Special School. The school also runs a two year vocational training programme in, for example, thanka paining, carpet making, tailoring and decorative drawing.

While all classes, except Tibetan language, are taught in either oral Chinese and/or CSL-supported oral Chinese, students amongst themselves mainly sign a mixed form of various CSL dialects and use only a few, or no TSL loans. Only some have had exposure to TSL as an extracurricular subject, when between 2001 and 2012 TDA volunteers offered classes at the school. This had not been the case in the Lhasa Special School since it moved to the outskirts of Lhasa, to the new and so-called "education district" in the summer of 2016. In the other 4 Special Schools established in other parts of the TAR, there is typically 
less signed and more oral instruction than at the Lhasa Special School, and the teachers of Tibetan language as a subject have even less TSL skills and resources.

In 2016/2017 students in the Tibetan language classes at the Lhasa Special School benefitted from the TSL manual alphabet that had initially been introduced by the TDA volunteers. It had quickly become used by the first government Tibetan language teacher, a dedicated Tibetan woman. As member of the pioneering group of teachers who started the Lhasa Special school and in charge of Tibetan language teaching, she also created a set of 100 new grammar signs in about 2001/2002, in order to represent specific spoken and written Tibetan grammatical terms and concept that she had to teach the students, as the school followed the regular Tibetan language programme and textbooks for all Lhasa schools. She used these with her students in the classroom and then created resources and shared them with other Tibetan language teachers, as the school began to grow and she was no longer the only Tibetan language teacher. ${ }^{7}$ The TSL manual alphabet and the Tibetan grammar signs were the only two, aspects of TSL used by the hearing Tibetan language teachers in the classes I attended in 2016 and 2017. With exception of the pioneering teacher who had also learned other TSL signs and used "spontaneous sign" to great effect, most of them used spoken Tibetan as a means of instruction, interspersed with the TSL manual alphabet and some grammar signs. The fluency in TSL finger spelling and grammar signs varied greatly between the most experienced teacher and others who had started teaching Tibetan language to deaf students more recently.

Overall 150 deaf students have graduated from the Lhasa Special School, of whom about 40 have gone on to a specialized high school (i.e. upper middle school), set of up for teaching deaf students in China proper. Out of these, just over ten had succeeded in entering colleges that are open to include deaf students as well as offering some CSL interpretion and/or captioning services.

The overall group of about 40 deaf Tibetans who had travelled and studied in China proper, have been profoundly exposed to the "special education-variety of CSL" (used mainly by teachers) as well as native CSL varieties - depending on where they went and the extent of their social networks. Nanjing and Shanghai sign varieties (Yang 2015) were most dominant among these Tibetan deaf students and graduates. Five graduates from these Chinese colleges have now been

7 Only 15 out of the 100 grammar signs are documented in the Standard Tibetan Sign Language Dictionary (TDA 2011: 481-485). They have all not been video recorded and as of 2017 most of them had fallen out of use. 
employed as teachers in the Lhasa Special School, using and spreading these varieties at the Lhasa Special School.

In Lhasa, there was initially little impact of the Special School students' primary use of CSL varieties on the TSL-dominant signers outside of the boarding school, as there was little to no contact (except during the extracurricular Tibetan and TSL classes). From about 2012 on, however, when the first deaf graduates from the local Special School who had gone on to high school and/or college in China proper returned to Lhasa again and started looking for work, they began to meet other deaf Tibetans. For instance in the TDA office, local tea and coffee houses, Lhasa tailoring shops, and an all-deaf incense workshop, and the sporadically organized TSL courses and Sunday Deaf Club meetings. It is most likely at this point that the phenomenon of mixing Chinese and Tibetan sign languages began to occur.

\section{4 „Spontaneous sign“ as a third phenomenon in the mix}

Deaf Tibetans often explained to me that they share a pool of body language, gestures and signs, which they refer to as "rang-jung lak-da", or "spontaneous sign language". They hold that some of this repertoire is shared with what linguists refer to as co-speech gestures of hearing Tibetans, such as the Tibetan language teacher at the Lhasa Special School mentioned earlier. In several interviews people estimated that "spontaneous sign" makes up approximately $30 \%$ (some even said $80 \%$ ) of the sign lexicon of what eventually became TSL. I observed how "spontaneous sign" was also drawn on in interactions between predominantly TSL and CSL Tibetan signers (Hofer 2019). A phenomenon similar to "spontaneous sign" has also been reported from Nepal, locally referred to as "natural sign” as analysed by Green (2014), and from Port Morsby, Papua New Guinea, locally referred to as "culture sign” and researched by Reed (forthcoming).

Many sign linguists hold that the number of shared signs between signers of different sign languages is larger as compared to that of words between two different spoken languages due to the higher iconicity of many sign languages (e.g. Guerra Currie et al. 2002). This has also been contested however (Taub 2004). 
Be that as it may, in Lhasa and the specific socio-linguistic context of deaf TSL signers and their deaf and hearing networks, "spontaneous sign” mattered.

This is a context of an only recently formalized local sign language, as explained above, that still features great variation. There has been a historical lack of formal schooling for deaf children and sign language interpreters do not exist. The use of TSL is also restricted to informal domains (for example, there is no TSL on TV and no TSL-interpreted public events) and the number of TSL signers is overall small. The time span has also been shorter and the spaces where the language has been used few, when compared with the vast breath of application and long-standing history of national European sign languages, such as French sign language (LSF), which is documented as early as the mid-18th century.

The existence of "spontaneous sign" in Lhasa means that unimodal codeswitching does not only take place in the local varieties of Tibetan and Chinese sign language, but that deaf Tibetans also draw on a pool of signs, gestures and bodily communication that is shared by all Lhasa Tibetans regardless of their dominant language modality. Spontaneous sign should therefore be considered a phenomenon of a third type and its existence has implications for communication across often too rigidly conceived deaf-hearing, Tibetan-Chinese and TSL-CSL social and linguistic boundaries.

\section{5 „Neither-goat-nor-sheep-sign“: Ideals of language purism and practices of sign mixing}

During a video-call TSL signers Drolma, Tashi, and myself were sitting in a Western-style café, ${ }^{8}$ talking with Wangchen, a competent TSL signer and acquaintance. After some time, Drolma signed to Wangchen, "Have you forgotten your Tibetan signs? Now you sign 'thanks' in CSL." Tashi signed, "That's right, you do", himself also using a common CSL sign meaning "correct/right", instead of the TSL equivalent, which maps onto the affirmative "dug/red" ("you do/you have") in spoken Tibetan. Wangchen was caught a little by surprise, laughed and then commented, "Our signing is now a mix of Tibetan and Chinese signing, right?"

This conversation reveals that Tashi and Wangchen do shift to and borrow CSL signs in an otherwise TSL-based conversation, even when Drolma and Tashi told me that they prefer to use Tibetan sign. And indeed, based on my notes and

8 All personal names used in this article are pseudonyms. Location names have also in some cases been changed to protect the anonymity of the research participants. 
a preliminary transcription of video-recorded conversations in which they chat with other TSL-dominant signers, there are few occurrences of code-switching to CSL. For example, they frequently use the CSL sign "to marry" and Drolma used to mouth or voice the Chinese term laoshe ("teacher") even when signing TSL for "teacher", thus creating a new code-blend involving a Tibetan sign and a spoken Chinese term. They did however not use CSL for the days of the week, which was otherwise common among TSL signers, or CSL numerals, which was also common. Their relatively "pure” TSL (at least as far as imports from CSL were concerned) might be explained by their limited contact with graduates from the Lhasa Special School thus far and their signing and mingling mainly with others who had been involved in the TSL project and of a similar age group. All of the Tibetan interlocutors in our video call, had known each other from the Sunday Deaf Club activities and other TDA-HI collaborative projects, in which Drolma had been involved since 2000, Wangchen since soon after and Tashi, who is also a bit younger, since 2009. At first encounter with TSL, Drolma and Wangchen were in their early and late 20s respectively, had minimal schooling and both were already too old to attend and study at the newly set-up Lhasa Special School. Tashi had grown up in a small village outside of Lhasa.

Among Drolma's close friends is Yangzom. After a few years of a joint informal play group at a local Lhasa hospital, they were reunited during the TSL project in the early 2000s, where they also gained some basic literacy in Chinese and Tibetan. They see each other for celebrations, such as Tibetan New Year, and get together in their spare time. Discussing the topic of mixing Tibetan and Chinese sign, Yangzom says she prefers and signs better in TSL. She added that it now surprised her that communication even with her long-standing friend Dekyi gets a little stuck sometimes, due to Dekyi's pervasive use of CSL signs. These remarks were made before Yangzom started a new job in an all-deaf sewing workshop, where a majority of her colleagues are CSL-dominant signers and she has gotten used to it since.

Dekyi's increased use of CSL signs is, despite her having been instrumental in completing the Standard Tibetan Sign Language Dictionary (Henceforth, for short, The Standard Dictionary) and serving as one of the functionaries of the TDA. Dekyi loves the Tibetan language and is one of relatively few deaf Tibetans who can read, write and also speak Tibetan well; she is also proficient in correctly fingerspelling Tibetan words using the TSL manual alphabet. Prior to losing most of her hearing as a teenager, she gained a solid Tibetan and Chinese language education at a regular school. After she was no longer allowed by the teachers to attend this school, she stayed at her family home before eventually moving to Lhasa and joining the TDA. She considers herself (and is considered by others) a deaf person (mi on-pa). Actively involved in developing TSL, Dekyi has praised 
Tibetan Sign Language, in a project funding application in written Tibetan, as a "butter lamp that shines a light in the darkness for those who lost their hearing or cannot speak", the butter lamp a salient metaphor for not only shining a light in the dark, but also accrueing religious merit.

Dekyi was the first to mention the term ra-ma-luk lak-da, the "neither-goatnor-sheep sign language" to me, back in January 2016, when we were discussing trends in sign language use among deaf people in Lhasa. At that point there was no sign for mixing Tibetan and Chinese sign, but the practice was already common. While dedicated to the value and the promotion of TSL, Dekyi often mocked herself and other Tibetans as becoming "neither-goat-nor-sheep" in their signing, herself using the spoken Tibetan word and thus participating in the same discourse pertaining to Chinese codeswitching in Lhasa Tibetan that is widespread among hearing Tibetans (cf. Tournadre 2003, Yeshe 2008).

Although from our conversations I knew that Dekyi had a personal preference for a purer TSL (in her terms, “only TSL”), as a functionary of the TDA I observed that she had to communicate with everybody, no matter which sign language or form of communication was required. The TDA is meant to represent "all” deaf Tibetans. ${ }^{9}$ For getting the work of the organization done the TDA had to hire graduates from the Special School, preferably those who had come back from China proper with college education. Working with these CSL-dominant signers, Dekyi has acquired a robust CSL repertoire and she now uses many CSL signs as part of her communication, even with those she used to sign mainly in TSL before.

While the two friends Drolma and Yangzom's longing and preferences for a purer TSL matter to them personally and have also seemingly influenced their choices of close friends and how they sign with them, Dekyi's opinions are more than a matter of personal choice and interest. Given her role in the TDA, she is required to balance working for deaf Tibetans' well-being and "development" all over the TAR (including Tibetans for whom TSL may not be the main sign language), yet also is responsible for the transmission of TSL into the future, a key mission of her organization. To pursue the latter has become difficult, however, especially since summer 2016 when the PRC government's State Plan for Sign Language and Braille Standardization Movement (2015-2020) (from now on, for short, the State Plan, CDPF et al. 2015) came to the knowledge of the TDA in Lhasa.

9 Note that this in fact only includes deaf Tibetans in the TAR and not Tibetans in other parts of China. To my knowledge there have not been any Han Chinese attendees to TDA activities. In terms of actual membership the numbers I have been given were vague. Perhaps the best indicator of a possible number of TDA members, was the 200 group members in 2016 of a Weixin (WeChat) group that the TDA had created to inform deaf Tibetans of its activities. 
The State Plan is to "promote the standardization (C. gui fan hua) of sign language" and "speeding up the research and creation of the state's common (C. tong yong) sign language and Braille” (CDPF et al. 2015: 4). Although this policy paper should technically not affect Tibetan Sign Language, its status, use and future development (since it is a linguistically different sign language from the national CSL addressed in the document), the State Plan and the policy of unification, have been used by TDPF officials in a way that implies that TSL is a part of the "national sign language" that the State Plan sets out to regulate and standardize. In line with this (mis)interpretation (likely to be related to a lack of commitment and imagination, and/or the political sensitivities attributed by the authorities to the Tibetan language and culture nexus), the TDPF had in 2017 ceased to fund TSL-related activities, including TSL language courses and materials. Instead, it promoted Tibetans' learning of CSL and gives permission to deaf Tibetans to contribute to research projects in China proper, which dedicate themselves to the creation or expansion of CSL corpora, for example, by documenting TSL lexical items or contributing Tibetan signs to otherwise CSL-research based outputs. The exact practices and projects that have begun in relation to this the state "common sign language" and its aims and objectives are currently not well documented.

Many of the TSL signers who had been actively involved with the TDA activities see the codeswitching of TSL and CSL in the form of what Dekyi calls a "neither-goatnor-sheep sign language" as something negative that ideally should be avoided. This ideology resonates with wider discourses and concerns over the influence of Chinese on the Tibetan languages and the region more broadly (Robin 2014) and with regard to Chinese codeswitching in modern Lhasa Tibetan (Yeshe 2008).

Yet, the group of people discussed in this section still engage in the practice, especially due to their expanding engagements with the CSL-dominant graduates from the Special School through work and friendships. Pragmatism and the necessities of communicating in daily life, as well as the lack of support for TSL within and outside of the Special School, make many people's idealistic positions untenable in practice in certain contexts. We can see clearly the tensions between many Tibetans' stated preferences, i.e. their language ideologies, and their linguistic behavior. These tensions also remind us as researchers that we should not make the assumption that people's attitudes result in corresponding linguistic behaviors.

This point resonates with Zeshan and Panda's study of bilingual signers, who found that the four study participants' language attitudes towards mixing two sign languages (two felt keeping the sign languages separate was prefera- 
ble, while the others supported the idea of mixing sign languages in the same stretch of discourse), had no bearing on their actual codeswitching practices as recorded on video (2015). There are also numerous studies on local sign language users' attitudes towards imports and influences from a globalizing American Sign Language (ASL) and national sign languages and to what extent these attitudes translate into their own linguistic behaviors and use of ASL (Cooper 2016, Moges 2016, Moriarty Harrelson, 2017, Nonaka 2014, Schmaling 2003).

In summary, the signers' attitudes discussed so far vary, all operate within a model that Dekyi termed the "neither-goat-nor-sheep" model of mixing languages - a model that implies the traditionaly negative connotations of something or someone being "neither-nor". The term she used - both in speech and sign - were still Tibetan terms and metaphors, close in meaning to hearing Lhasa Tibetans using the term. This was however not the only way to perceive and interpret the mixing of TSL and CSL.

\section{6 "A-bird-with-two-heads": Pragmatism and translanguaging}

Wangchen a TSL teacher and involved in various TSL project activities, holds different opinions on the mixing of TSL and CSL from those analyzed in the previous section and expressed by his peers. At home Wangchen signs with his deaf wife in TSL with the usual CSL loans and occasional switches to CSL signs, even when there is a TSL equivalent. While I observed that his wife tends to speak Tibetan with their hearing son, Wangchen signed a more basic form of TSL with him. ${ }^{10}$ Outside of the home, he has built many friendships and working relations with deaf people associated with the TDA and the TSL project, as well as with those who have graduated from the Special School. Based on a preliminary review of my video recordings and from what I observed during fieldwork, he uses a larger number of CSL signs with CSL-signing Tibetans, than with his wife and son or TSL-dominant friends. Regardless, most signers perceive him as one of the most expert and fluent signers in TSL.

10 He also lately has made more effort to voice Tibetan expressions alongside his signing (which he was not used to and also found very difficult), as his son's teachers expressed concerns to them about their son's slow speech development. For the time being, they had decided to prioritize speech over sign. 
We also discussed the topic of mixing Tibetan and Chinese signs. In such a context I once explained to him the Tibetan term ra-ma-luk lak-da, "neither-goatnor-sheep sign," after Dekyi had first mentioned it to me to capture the TSL-CSL mixing. I explained how ra-ma-luk was commonly used for things and people that are neither/nor and that the term is now commonly used for the mixing of spoken Tibetan and Chinese. He didn't know that expression and it took me some time to explain it accurately in TSL, as there was not yet a sign to denote such a practice. Once I had finished my explanation, Wangchen responded that this concept did not quite fit the case of Tibetan signers' mixing of Tibetan and Chinese signs. They are more like " $a$ bird with two heads", one, he said, was a Chinese "sign head" and the other a Tibetan one. Depending on the situation, one would use one and/or the other, often in quick succession.

For Wangchen the increasing mixing of TSL and CSL was a natural development and it was most important to him to be able to communicate - no matter how. He gave some examples: "Say you want to sign knife but you don't know the Tibetan sign for it, you use the Chinese one. Or for pizza, the same." I asked, "Do you mind this mixing?” He replied, “No, it doesn't matter - it's like this: we meet someone and we sign. And when I do not have a sign to explain something, or the other person doesn't understand a sign I use, I pick another sign for it and then that person understands - so it is very useful.” Although in reality Wangchen signs many CSL signs for which he has TSL signs and for which others would understand the TSL sign, the underlying ideology he expresses seems to be that the primary goal of communication is understanding each other. For him this means that communication happens in sign, as he is deaf, has little speech and like all of his peers, uses no hearing aids or implants. Given how relatively few Tibetan signers there are in Lhasa altogether, he thinks people should be able to communicate within that wider group of deaf people, irrespective of their preferred sign languages or educational background.

While thinking less than Dekyi about the implications of mixing TSL and CSL for the future of TSL, Wangchen shares with her the outlook and responsibility to communicate with a wide net of deaf Tibetans due to their TDA work and as TSL teachers. They also share a sense that it is urgent to bring deaf Tibetans together as much as possible, whatever form of communication this takes, so that deaf people can support each other and improve their livelihoods. This concern found expression in adding a CSL component towards the end of the most recent TSL course taught in Lhasa in November 2016, after a suggestion by the TDA leadership. ${ }^{11}$

11 The CSL module was taught by a graduate of the Lhasa Special School. 
During a more formal autobiographical interview in February 2016, I asked Wangchen about his thoughts on the future of TSL. "Tibetan sign is very useful. [They] should go together. We should not just develop and use one [i.e. CSL] and leave Tibetan sign aside. Chinese sign can be a real headache, with the fingerspelling of Pinyin terms and all. Many [Tibetans] find them very hard to understand. It's very different from our sign language, which has strong and many spontaneous expressions and which can be understood by all and easily. It makes [people] happy, they like [using] it.” I asked, “And what do you think would need to be done to strengthen Tibetan sign?" He said "We need to collect and create many more signs - in fact we need to have signs for everything. Now we have only about 1200, but we need many more in the future. We need signs for all the Tibetan words. That's what I think would be important. And then we need to teach this full repertoire more widely."

In this last statement he seems to suggest, as some examples in the literature on ideologies of users of other young sign languages do (e.g. Hoffmann-Dilloway 2008, 2016), that a signed language should have signed equivalents of all the words of the surrounding spoken language of the area or ethnic group. And, furthermore, that "signs" should be recorded in written language documents and formally taught, rather than understood as used and simply spread through use in an adult signing population.

Wangchen's use of the metaphor of a "bird-with-two-sign-heads" suggests that he does not support a neither-nor or either-or, but rather an and-and perspective on the mixing of TSL and CSL. He also expresses a love for "spontaneous sign", which he says is easily understandable by deaf Tibetans, at times even by hearing people. When asked directly, Wangchen expressed his concerns about the future of TSL. His ideas for developing Tibetan signs suggest a way to strengthen the bird's Tibetan "sign head," as if this were a prerequisite for this bird's head to be used as much as its Chinese "sign head". His comments suggest that there needs to be a better balance to the current much greater official support for all kinds of Chinese language in the TAR, through more support for Tibetan languages, whether signed, spoken or written. Put simply, in many settings, especially education and other government-related domains, Tibetans have no longer a choice as to which language(s) to use, with a growing tendency to Chinese in all public spaces in Lhasa (except monasteries, nunneries and some teahouses) and Tibetan relegated to Tibetan homes and 8 hours of obligatory Tibetan language classes a week for students in primary school. Therefore, only time will tell what the results of Wangchen's fluent adaptation to contexts and communication partners will be 
like. It also remains open what the development of further Tibetan Sign Language materials that he envisions, will look like and to what extent these might actually impact linguistic behavior of deaf Tibetans and their codeswitching to CSL. There seems to be some overlap of his opinions with other signers discussed below, whose new sign of the "goat-and-sheep language" (rather than "neither-goat-norsheep sign") seems to be closer to his perspective, than to the spoken and written Tibetan expression "neither-goat-nor-sheep sign".

\section{GOAT-SHEEP-SIGN-MIXED: Language hierarchies, national orientations and code-blending}

A large group of adult Tibetan signers in Lhasa sign almost exclusively in the local variant of CSL, due to their exposure to and adoption of CSL at the Lhasa Special School. Here Chinese and CSL dominate and little spoken/written Tibetan or TSL are taught, known or used by the students. Some of these signers, as mentioned earlier, were further exposed to CSL and its variants during their studies at high school and College in China proper. I only include in the discussion here those who have already graduated from that school and now live in Lhasa or surroundings. Those currently attending the Lhasa Special School have had almost no opportunity to come in contact with TSL signers, apart from learning and using the TSL manual alphabet and the grammar signs during formal Tibetan language classes, as well as using aspects of "spontaneous sign."

Among the graduates from the Special School, the majority are not aware of, or discuss their non-use of TSL. Those whom I asked, do not seem concerned about only relying on CSL. Rather, they see use of CSL as an expression of their cosmopolitan and national outlook, welcoming what they see as CSL's greater lexical repertoire as well as the more advanced educational opportunities that can be accessed via CSL by deaf people in China proper. They also hope to embrace professional openings (such as teacher posts) within the TAR's now five Special Schools, which require CSL and Chinese (TSL is no requirement at all, even for Tibetan language instructors). Many among this group are also simply more familiar and comfortable with CSL and have taken "deaf turns" (Friedner 2015) earlier in their lives than many in the TSL-dominant group.

Three of the so-far five deaf Tibetan teachers employed by the TAR's Special Schools fall into this category. To them, CSL opened up unprecedented educational opportunities, such as attending technical colleges (xué yuan) in Beijing, Nanjing, Tianjin, Xian and Shanghai, and they have gained great competence 
in (several varieties of) CSL. ${ }^{12}$ Despite the pervasive use of CSL in this group of signers, the deaf Special School teachers and several others claim that they use Tibetan "spontaneous sign" and TSL for Tibetan concepts that "do not exist in CSL": Tibetan clothes and food items, for example, such as a chuba (Tibetan robe) or butter tea. However, my own observations and video recordings do not bear this out. Rather, I have seen them describe such items in CSL (for example for chuba they sign "Tibetan dress") and they have their own local variety of CSL sign for butter tea (literally signing "oil drink"). Even for place names in Tibet, for "Tibet" and "Tibetans", and for "China" and "Chinese" they tend to use CSL signs, which tend to incorporate a Pinyin fingerspelling component related to the romanization of Chinese characters (Hofer, forthcoming). This is significant as these signs thus might become removed from cultural and political sensibilities shared by many Tibetans, especially with regard to signs for Tibetan places and the Tibetan people (cf. Hofer 2016).

Some among the Lhasa Special School graduates view their lack of TSL more critically, and with regret even. Among them are Tenzin and Gendun, who over a joint lunch in the Barkor area of central Lhasa commented on their conundrum. At the school they had been exposed to the extra-curricular TSL classes on Saturdays (cf. Hofer and Sagli 2017), which Tenzin in particular enjoyed. But she said that whenever they attempted to use their newly-learned TSL in the regular classes, their hearing teachers (at that time there were no deaf teachers) would correct them and instruct them to use CSL instead. Tenzin's fingerspelling of Tibetan was still good, and perhaps due to her apprenticing as a thanka (Buddhist scroll) painter she also knew many TSL signs for the various Buddhas and Bodhisattvas of the Tibetan Buddhist pantheon, the main icons of Tibetan thanka paintings. Gendun expressed similar feelings. While both said they would love to use more TSL, they found little space to use and practice it. Neither of them were involved or even aware of the then-ongoing TSL classes and occasional Sunday Deaf Club activities organized by the TDA. At the time of writing, Tenzin had stopped painting thanka and Gendun was hired as an assistant teacher to the Lhasa Special School. Given the school's language policy and him being reunited with other CSL-signing deaf and hearing teachers, he does not feel in a position to expand his use of TSL.

The opinions and ideologies of some other CSL-signing Tibetans were not clear cut and oscillated between an appreciation for the cosmopolitan potential

12 The use of a national sign language or even a foreign sign language such as American Sign Language in national deaf education is common in many other places too and often thought by teachers to open up educational opportunities (Kusters 2014; Schmaling 2003). 
of, as well as familiarity with CSL, and also a sense of regret for lacking TSL. Two women stood out among this group. Both had graduated from the Special School. Lhamo had obtained a College degree from a university in China proper in 2015 and almost immediately landed a job at one of the newly established Special School outside of Lhasa. Khandro meanwhile completed a vocational training and subsequently married, shortly afterwards having a child. Then she began to work at the TDA. Both of them were close friends of Dekyi, the aforementioned TDA functionary. While Khandro's CSL and that of another work colleague had definitely instigated TSL-dominant signers at the TDA to pick up more CSL, as already discussed, both Khandro and Lhamo wanted to learn more TSL from others to expand their repertoire.

That said, when I asked Lhamo what she thought the future of communication among deaf Tibetans, she gave a pragmatic answer. As if I didn't know, she said with slight surprise: "TSL alone is not enough, there are not enough signs. We need Chinese sign, as they have a sign for everything." We discussed this for a while, myself being well aware that these arguments had been also made in favor of Chinese language instruction in mainstream, Lhasa schools, and against the use of Tibetan in education. Lhamo continued: "Look, Tibetans use so much Chinese nowadays, even for technical things where we have Tibetan terms. The same in sign language, why not use the Chinese signs for terms we don't have?" She thought a blend of the two sign languages would be the best, using the spoken term "neither-goat-nor-sheep sign language" in a self-conscious and not inherently negative way. At the end of that conversation, she confirmed her opinion: "Yes, to mix and blend the two is best, use Chinese and Tibetan sign, mixed together."

Lhamo's argument, and also Wangchen's comments above, that there were insufficient Tibetan signs overlaps with the sign language ideologies held by several hearing teachers and the leadership of the Lhasa Special School, who in this way explained and defended the use of CSL as a medium of instruction next to Chinese to me. They were, however, often uninformed about not only recorded but also the actual extent of the TSL lexicon. For example, one long-established teacher wrongly thought there were only 400 TSL signs ${ }^{13}$. They also did not know about the interest expressed by the TDA in producing more volumes of The Standard Dictionary to further document the TSL repertoire. The ideology that more

13 The Standard Dictionary has overall 1,437 sign entries. A Nanjing-based research project with four fluent TSL signers from Lhasa in 2012 and in 2014 found and collected a combined TSL lexicon of 6300 non-CSL TSL signs (personal communication, November 2016). What counted as "one" sign here is not known to me. 
documented words and signs are better, resonates with ideologies in many other places about the status of the respective languages and their users.

Given the wider Chinese-medium instruction in Lhasa schools, it would have been exceptional for the Lhasa Special School to use TSL as a medium of instruction, not least because this would have had to involve a collaboration with the TDA. As discussed above the TDA was affiliated until 2014 with Handicap International, and working with an INGO had become particularly sensitive and problematic for Tibetans after a new series of protests began in 2008.

Several CSL-dominant Tibetans seem to advocate a form of translanguaging, which has been defined as "the deployment of a speaker's full linguistic repertoire without regard for watchful adherence to the socially and politically defined boundaries of named (and usually national and state) languages" (Otheguy, Garcia and Reid 2015:283). Dekyi earlier on called this by the spoken Tibetan term "neither-goat-nor-sheep sign", a term also used in spoken form by Khandro in the past. They both did not sign the term, as both became deaf in their teens and they speaks fluent Tibetan. And yet, at the very end of a stint of fieldwork, in late June 2017, Khandro and Dekyi were experimenting with and showed me new signs for this linguistic practice ${ }^{14}$ : they signed RA-LUK-LAK-DA ("goat-sheep sign") and RA-LUK-LAK-DRÉ ("goat-sheep-sign-mixed"). Rather than the neithernor model and its claims to purity of either the goat or the sheep language, these signs, like Wangchen's two-headed bird, confidently refer to the blending and co-use of Tibetan and Chinese sign. What is more, the latter sign mixes TSL and CSL: "goat" is signed the same way in TSL and CSL, so it's hard to know which, but, "sheep" is different in the two languages and they used the CSL sign. "Sign" and "mixed" were both TSL signs. This compound sign subsequently has been used among several signers of this group when discussing the mixing of the two sign languages with me (see Figure 2) and "Chinese-Tibetan-mixed sign" has also come into use (Figure 3).

14 This was the first time I saw a Tibetan person sign this term. 

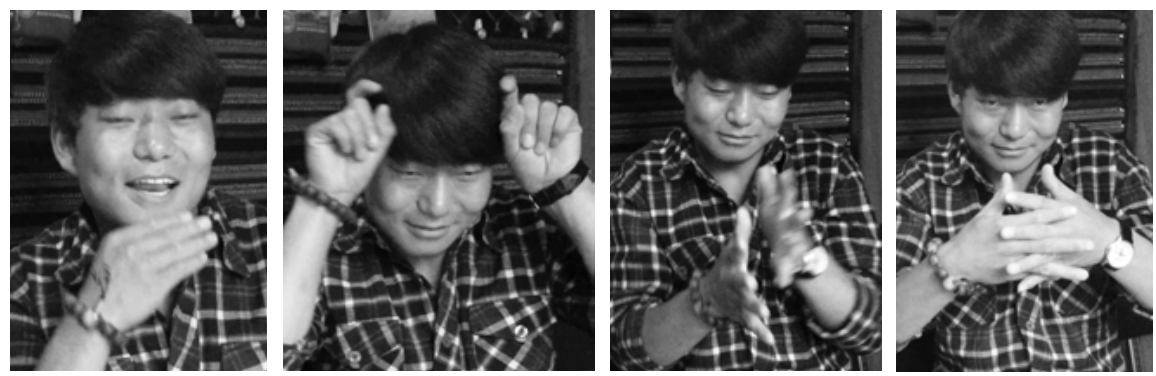

Figure 2. RA-LUK-LAK-DRÉ (GOAT-SHEEP-SIGN-MIXED).
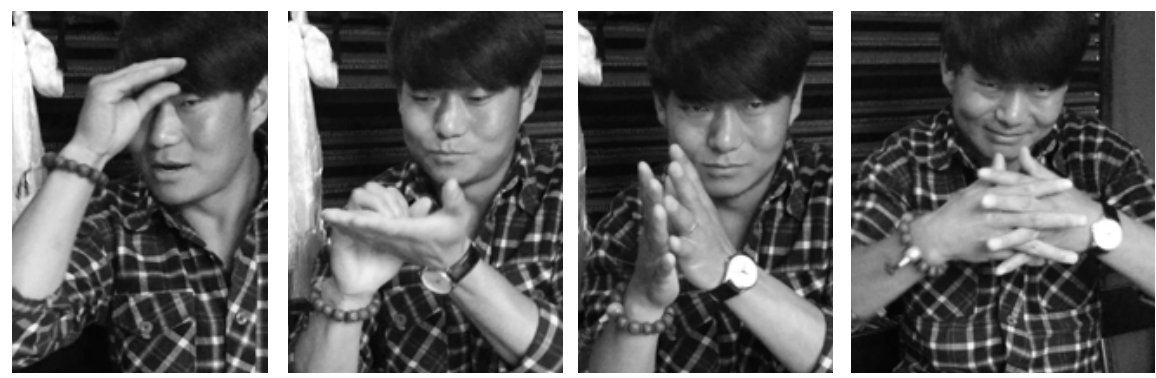

Figure 3. GYA-BÖ-LAK-DRÉ (CHINESE-TIBETAN-SIGN-MIXED).

Overall, the sign "goat-and-sheep-sign-mixed" and indeed most of the discussion of mixed signing, focuses on the lexicon, not syntax or other aspects of grammar, with the exception of a CSL-dominant signer who pointed also to aspects of mixing of TSL and CSL grammar. The origin and implications of my research participants' ideological focus on the level of words/signs needs to be further researched. It might well have to do with the legacy of the TSL project, as almost all of the TDA's efforts so far focused on lexicon and dictionaries as a means to standardize, formalize and promote Tibetan Sign Language. Erika Hoffman-Dilloway's work in Nepal discusses a similar focus in the process of standardizing Nepali Sign Language (2008). Also relevant may be the initial mode of transmission of CSL to the students of the Lhasa Special School via hearing Tibetan teachers who encountered and studied CSL mainly through a standard two-volume CSL dictionary, rather than through everyday communication with deaf Chinese people. 


\section{Conclusion}

Chinese and CSL are promoted top-down as the nominal "national languages" of the deaf in China through the country-wide Special School system. New policies proposed through the State Plan (CDPF 2015) and related documents further support this. This is even though CSL is not officially recognized as one of the minority languages of the PRC (Huang and Gu 2014, Yang 2015). The promotion of Chinese and CSL takes place regardless of the location of a Special School or the group of deaf people it may cater to in an ethnic minority areas (such as the TAR), where other spoken minority languages are used and are official languages. Central government and official language ideologies claim to thus offer better educational opportunities for all deaf people in China. Yet, as this chapter has discussed, these ideologies collide with the aspirations, ideologies and, at times, linguistic behaviors of some deaf Tibetans - in particular those who had actively worked in the TDA in support of TSL and had considered it the local equivalent of the "father tongue" (i.e. the native language) of all deaf Tibetans. Yet, the official ideologies in support of CSL do not collide with other deaf Tibetans, like those who are CSL-dominant, thus offering a complex socio-linguistic picture.

The diverse sign language ideologies that I have discussed here have emerged and changed over the past years in relation to earlier (now defunct) collaboration of a group of locals with the INGO Handicap International, the founding and activities of the TDA, the increasing interactions among deaf signers in an urban environment, the founding of the TAR Special Schools, and domestic mobility of some deaf Tibetans enabling them to go to China proper. These ideologies are found among, but do not map onto neatly, two groups of deaf Tibetan signers in Lhasa, whom I have referred to as TSL-dominant and CSL-dominant. Even these classifications may be inadequate, however. In the written words of one interlocutor, even the "father tongue of deaf Tibetans is now neither-goat-nor-sheep" (rang-ki pha-ké lak-da de yang ra-ma-luk). Deaf Tibetans have been referring to the resulting language practices with the spoken and written Tibetan terms "neither-goat-nor-sheep-sign" and "mixed-sign-language”, as well as by new signs that render this practice as "goat-sheep-mixed-sign", or "Chinese-Tibetan-mixed sign" (Figure 2 and 3). Mixing CSL lexical items into the communication of TSL-dominant signers has become particularly common, while comparatively fewer CSL-dominant Tibetan signers codeswitch to or import loans from TSL into CSL. This imbalance is partly due to local social dynamics, the influence of powerful state institutions and state-led sign language ideologies that aim to tune down regional or ethno-linguistic differences within the PRC to instead promote national (political) unity and exert cultural and linguistic control homogeneity. 
The broader tensions and political sensitivities over Tibetan language, culture and political self-representation are additional important contextual influences.

Crystal (2000) and Myers-Scotton (2007: 78-79) have suggested a three stage linguistic process leading to the loss of spoken minority languages world-wide: the primary use of the minority language being followed by an emerging bilingualism, which then quickly declines and leads - usually through the younger generation's use and preference for the new language - to fewer and fewer people using their "old" minority language (see also Thomason 2015: 12). Most sign languages, however, are not used in the families into which deaf people were born and the linguistic developments among deaf signers, at least in Lhasa, seem to follow a different path. The "new language" here (i.e. CSL) is actually the "old" and "native" language of the Tibetan Special School students, while those who first encountered TSL as their "father sign language" (pha-ké lak-da) do not actually use TSL wholesale, but incorporate large numbers of CSL lexical items into TSL grammatical structure through either loans or codeswitching. What will come after this current stage, only time, and the ongoing documentation of deaf Tibetans' communication and their social, professional and individual lives, will be able to tell.

In Lhasa, different attitudes towards the mixing of Tibetan and Chinese sign, as well as towards "spontaneous sign" shared between all deaf Tibetans (and to some extent their hearing teachers, family members and others), are unevenly distributed and they do not necessarily influence the respective linguistic behaviors of the very same people who hold them. Furthermore, both language ideologies as well as linguistic practices are changing in response to ongoing wider societal changes in Lhasa, in Tibet and in the PRC, specifically with regard to a changing legal environment for TSL and CSL, language shift towards Chinese, and changing educational and professional opportunities encountered by deaf people.

The signs and the practices related to the mixing of TSL and CSL challenges assumptions about the bounded nature of local (sign) languages, as well as the social boundaries of those who use them. In addition to the actual linguistic behaviors, it might after all be the language attitudes and ideologies, and the contexts and social relations within which these are embedded, that establish significant social differentiation.

\section{References}

Adam, Robert. 2017. Unimodal bilingualism in the Deaf community: Language contact between two sign languages in Australia and the United Kingdom. London: University College London PhD dissertation. 
China Disabled People's Federation (CDPF); Education Bureau; State Language Commission and the State News, Publishing and Broadcasting Administration. 2015. State plan for sign language and Braille standardization movement (2015-2020). http://www.cdpf.org.cn/ yw/201512/t20151229_537798.shtml (accessed 13 August 2019)

Cooper, Audrey C. and Nguyen Tran Thuy Tien. 2016. Signed language community-researcher collaboration in Viet Nam: Challenging language Ideologies, creating social change. Journal of Linguistic Anthropology 25(2): 105-128.

Crystal, David. 1992. An encyclopedic dictionary of language and languages. Oxford: Blackwell. Crystal, David. 2000. Language death. Cambridge: Cambridge University Press.

Friedner, Michele. 2015. Valuing Deaf worlds in urban India. New Brunswick: Rutgers University Press.

Green, Elizabeth Mara. 2014. The Nature of Signs: Nepal's Deaf Society, Local Sign and the Production of Communicative Sociality. Berkeley: University of California PhD dissertation.

Guerra Currie, Anne-Marie, Richard P. Meier and Keith Walters. 2002. A cross-linguistic examination of the lexicons of four sign languages. In Richard P. Meier, Kearsy A. Cormier \& David G. Quinto-Pozos (eds.), Modality and structure in signed and spoken languages, 224-236. Cambridge: Cambridge University Press.

Hofer, Theresia. 2016. "Indexicality and Tibetanness through sign: Places and belonging” Paper presented at the Language, Indexicality and Belonging Linguistic Anthropology Conference at Somerville College, University of Oxford, 7-8 April.

Hofer, Theresia. 2017. Is Lhasa Tibetan sign language emerging, endangered, or both? International Journal for the Sociology of Language 245 (May): 113-145.

Hofer, Theresia. Forthcoming. Logics of Iconics: Place names in Tibetan Sign Language (TSL) and their Use among Signers in Lhasa. Minpaku Sign Language Studies, Vol 3, 2020, Japan, Minpaku.

Hofer, Theresia and Gry Sagli. 2017. 'Civilising' Deaf people in Tibet and Inner Mongolia: Governing linguistic, ethnic and bodily difference in China. Disability \& Society 32(4): 443-466.

Hoffmann-Dilloway, Erika. 2008. Metasemiotic regimentation in the standardization of Nepali Sign Language. Journal of Linguistic Anthropology 18(2): 192-213.

Huang Wei, and Gu Dingqian. 2014. Status quo of sign language. In Yuming Li and Wei Li (eds.) Language situation in China (Language policies and practices in China, Vol. 2). Berlin \& New York: Mouton de Gruyter, 43-50.

Kusters, Annelies. 2014. Language ideologies in the shared signing community of Adamorobe. Language in Society, Vol 43: 139-158.

Kusters, Annelies and Michelle Friedner. 2016. Introduction - DEAF-SAME and Difference in international Deaf spaces and encounters. In Michele Friedner and Annelies Kusters (eds.) It's a small world: International Deaf spaces and encounters. Washington DC: University of Gallaudet Press, p. ix-xxix.

Kroskrity, Paul. 2005. Language ideologies. In Alessandro Duranti (ed.) A Companion to linguistic anthropology. 496-517. http://www.blackwellreference.com/subscriber/book. html?id=g9781405144308_9781405144308 (accessed 8 May 2017).

Moges, Rezenet Tsegay. 2016. Challenging sign language lineages and geographies: The Case of Eritrean, Finnish, and Swedish sign languages. In Michele Friedner and Annelies Kusters (eds.) It's a small world: International deaf spaces and encounters. Washington DC: University of Gallaudet Press, 83-94. 
Moriarty Harrelson, Erin. 2017. Regimes of mobilities: Deaf development, NGOs and Deaf tourism in Cambodia. Washington DC: American University PhD dissertation.

Myers-Scotton, Carol. 1997. Dueling languages: Grammatical structure in codeswitching. Oxford: Clarendon Press.

Nonaka, Angela M. 2014. (Almost) Everyone here spoke Ban Khor sign language - until they started using TSL: Language shift and endangerment of a Thai village sign language." Language \& Communication 38:54-72.

Otheguy, Ricardo, Ofelia García, and Wallis Reid. 2015. Clarifying translanguaging and deconstructing named languages: A perspective from linguistics. Applied Linguistics Review 6(3):281-307.

Pavlenko, Aneta. 2007. Autobiographic narratives as data in applied linguistics. Applied Linguistics 28(2):163-188.

Plaza-Pust, Carolina and Esperanza Morales-López (eds.). 2008. Sign bilingualism: Language development, interaction, and maintenance in sign language contact situations. Amsterdam: John Benjamins Publishing Company.

Quinto-Pozos, David. 2000. Contact between Mexican sign language and American sign language. Austin: University of Texas PhD dissertation.

Reagan, Timothy G. 2010. Language policy and planning for sign languages. Washington, DC: Gallaudet University Press.

Reed, Lauren W. Forthcoming. „Culture sign is my favourite“: Bilingualism and identity in the Port Moresby deaf community, Papua New Guinea. For special issue of Asia-Pacific Language Variation Journal.

Robin, Françoise. 2014. Streets, Slogans and Screens: New paradigms for the Defense of the Tibetan Language. In: Trine Brox \& Ildikó Bellér-Hann (eds.) On the fringes of the harmonious society - Tibetans and Uyghurs in socialist China. Copenhagen: NIAS Press, 209-234.

Schmaling, Constanze. 2003. A for apple: The impact of western education and ASL on the Deaf community in Kano state, northern Nigeria. In: Leila Monaghan, Constanze Schmaling, Karen Nakamura, \& Graham H. Turner (eds.) Many ways to be Deaf: International variation in Deaf communities. Washington, DC: Gallaudet University Press, 302-310.

Taub, Sarah F. 2004. Language from the Body: Iconicity and metaphor in American sign language. Cambridge: Cambridge University Press.

TDA (Tibet Deaf Association). 2011. Bod kyi rgyun spyod lag brda'i tshig mdzod [Dictionary of everyday Tibetan sign language] (Chinese titlepage title: Longya shouyu zangyu cidian [Deaf Tibetan sign language dictionary], English title on title page: Standard Tibetan sign dictionary). Lhasa: Bod ljongs mi rigs dpe skrun khang [Tibet Minority Press].

TDPF (Tibet Disabled People's Federation) \& HI (Handicap International). 2002. 'On lkugs kyi lag brda'i bod yig tshig mdzod deb dang po [Alphabetical dictionary of Tibetan deaf-dumb sign language - Volume 1], (English title on title page: Tibetan sign language dictionary Volume I). Lhasa: Bod ljongs mi dmangs dpe skrun khang [Tibet People's Press].

TDPF (Tibet Disabled People's Federation) \& HI (Handicap International). 2005. Bod kyi lag brda'i slob deb - dang po [Tibetan sign language book - Volume 1]. Lhasa: Privately published.

Thomason, Sarah. 2015. Endangered languages: An introduction. Cambridge, Cambridge University Press.

Tournadre, Nicolas. 2003. The dynamics of Tibetan-Chinese bilingualism. China Perspectives 45 (January-February): 1-9. 
Yang, Junhui. 2015. Chinese sign language. In Julie Bakken Jespen et al. Sign Languages of the World: A Comparative Handbook. Berlin and Boston: De Gruyter, 177-194.

Yeshe, Kelsang. 2008. Chinese codeswitching in modern Lhasa Tibetan: A motive-driven linguistic behavior of Lhasa Tibetans. Oxford: University of Oxford DPhil dissertation.

Zeshan, Ulrike and Sibaji Panda. 2015. Two languages at hand: Code-switching in bilingual deaf signers. Sign Language \& Linguistics 18(1): 90-131. 
